\title{
LIGHT EMITTING FABRICS FOR PHOTODYNAMIC TREATMENT OF VULVAR PRIMARY EXTRAMAMMARY PAGET'S DISEASE
}

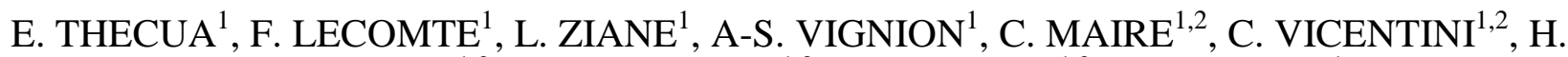 \\ ABIRACHED $^{1,2}$, D. STAUMONT ${ }^{1,2}$, L. MORTIER $^{1,2}$, S. MORDON $^{1}$. \\ ${ }^{1}$ Univ. Lille, Inserm, CHU Lille, U1189 - ONCO-THAI - Image Assisted Laser Therapy for \\ Oncology, Lille, France \\ ${ }^{2}$ Department of Dermatology, CHU Lille, Lille, France
}

\begin{abstract}
Primary Extramammary Paget's disease (EMPD) is a rare cancer that mainly affects the genital region including vulvar and perianal areas.

Without treatment, vulvar EMPD progresses and presents always more erythematous and pruritic plaques, which may become ulcerated and erosive. To control disease progression and symptoms usually experienced by patients, surgical excision is the mainstay of treatment. Unfortunately, even after large surgical excision with intra-operative margin control, recurrences are common [1]. For recurrent patients which undergo multiple resections, severe functional and sexual alterations are described.
\end{abstract}

Only few data are available on the efficacy of alternative conservative treatments, such as laser ablation, radiotherapy, topical chemotherapy and photodynamic therapy (PDT) [2]. To date, none of them can be considered as a solid alternative to surgical excision yet [3]. Nonetheless, multiple clinical cases suggest that PDT could provide the opportunity to treat subclinical lesions, and give some relief on patient's symptoms of the disease [4-6].

Unfortunately, the benefits of using photodynamic therapy for vulvar EMPD remains a challenge to demonstrate, because of the inhomogeneous illumination of vulvar and perianal areas, and the extreme pain that patients usually experienced during the illumination procedure that may lead to premature end of treatment $[7,8]$.

Resulting from the knowledge of previous works on actinic keratosis of the scalp treatment with PDT, light emitting fabrics (LEF) technology could address both of the hereinbefore described issues [9-12]. A new medical device based on LEF named PAGETEX® dedicated to illumination of vulvar and perianal areas has been developed. The device delivers a homogeneous red light $(635 \mathrm{~nm})$ with a low irradiance during $2 \mathrm{~h} 30$, for a total fluence of $12 \mathrm{~J} / \mathrm{cm}^{2}$.

The PAGETEX® device is being assessed in a clinical study (NCT03713203) which aims to establish PAGETEX®PDT efficacy and tolerability.

Keywords: Light emitting fabrics, textile, Extramammary Paget's disease, photodynamic therapy 


\section{INTRODUCTION}

Primary Extramammary Paget's disease (EMPD) of the vulva is a rare skin cancer that mainly affects the genital region of elderly female population. Patients develop red eczematous and pruriginous plaques with a chronic evolution. Common dermatological symptoms and the lack of knowledge of the Paget's disease often lead to late diagnosis. To control disease progression and symptoms usually experienced by patients, surgical excision is the mainstay of treatment. The excision can be a total vulvectomy, or be delimitated to the lesions with common margins of $2 \mathrm{~cm}$ in width and $0.5 \mathrm{~cm}$ in depth. However, recurrences are common (up to 58\% within 15 months to 14 years) [1], and recurrent patients suffer from severe functional and sexual alterations. Alternative treatments are studied like topical chemotherapy, laser ablation or radiotherapy but the adverse effects are numerous and the results are not enough superior to surgical excision. To date, none of these treatments can be considered as a solid alternative [3]. PDT is also studied [2, 4-6] but unfortunately, the benefits of using photodynamic therapy for vulvar EMPD remains a challenge to demonstrate, because of the inhomogeneous illumination of vulvar and perianal areas, and the extreme pain that patients usually experienced during the illumination procedure that may lead to premature end of treatment [7,8].

Resulting from the knowledge of previous works on actinic keratosis of the scalp treatment with PDT, light emitting fabrics (LEF) technology could address both of the hereinbefore described issues [9-12]. A new medical device based on LEF named PAGETEX® dedicated to illumination of vulvar and perianal areas has been developed.

\section{MATERIALS AND METHODS}

\subsection{Key points for PDT on EMPD}

In Europe, PDT has been approved for precancerous and cancerous lesions of the skin when the photosensitization is given by the topical application of methyl-amino levulinate (MAL) contained in the Metvix® cream (Galderma). Applied to the area to be treated, this pharmacological agent called precursor induces the specific accumulation of PS, Protoporphyrin IX (PpIX), in tumor cells.

The light source most commonly used in Europe to activate the PpIX is the Aktilite ${ }^{\circledR}$ CL 128 (Galderma SA). It results on a LED rigid panel that delivers a red light with a total light dose of $37 \mathrm{~J} / \mathrm{cm}^{2}$ in 8 to 10 min [13]. Although often described as very painful by patients, PDT remains one of the first line treatments, especially for the management of multiple actinic keratosis because it allows to treat large areas with a satisfactory response rate and excellent cosmetic results. Several recent studies [14-16] have shown that the use of a reduced dose and/or irradiance provide similar efficacy, with a limited pain.

Furthermore, classical light sources used for the photodynamic treatment are usually planar while the targeted surfaces are mainly curvy [17]. The development of flexible light sources, able to fit to human body shapes could overcome the hereinbefore described drawbacks.

\subsection{PAGETEX®: light emitting fabrics-based biophotonic device}

Developed in the framework of CIP PHOS-ISTOS European project, LEF technology consists in the integration of polymethyl methacrylate (PMMA) step index optical fibers (TORAY, Tokyo, JAPAN within a fabric structure during the knitting process. By controlling macrobendings and yarn tension during the knitting process, homogeneous light emission over the entire surface of the sample can be obtained [18, 19].

While coupled to any LASER source, the LEF-based devices result on a passive light emitting surface, just like frontal or cylindrical diffuser end optical fibers.

Several clinical studies using LEF for PDT of actinic keratosis have been published [15, 16] and launched industrialization of FLUXMEDICARE device (MDB TEXINOV, Saint Didier de La Tour, FRANCE). The good clinical outcomes obtained with light emitting fabrics-based devices have also motivated the development of a new device dedicated to the management of vulvar EMPD. The PAGETEX ${ }^{\circledR}$ device consists of a flexible light diffuser that embeds knitted light emitting fabrics with an efficient surface of $380 \mathrm{~cm}^{2}$ and an optical fiber, providing the connection with a $635 \mathrm{~nm}$ medical CE laser source. The PAGETEX® device is a source of non-laser optical radiation and provides 
diffused illumination for photodynamic therapy, and is a class IIa medical device in the European Medical Device Classification System (93/42/EEC).

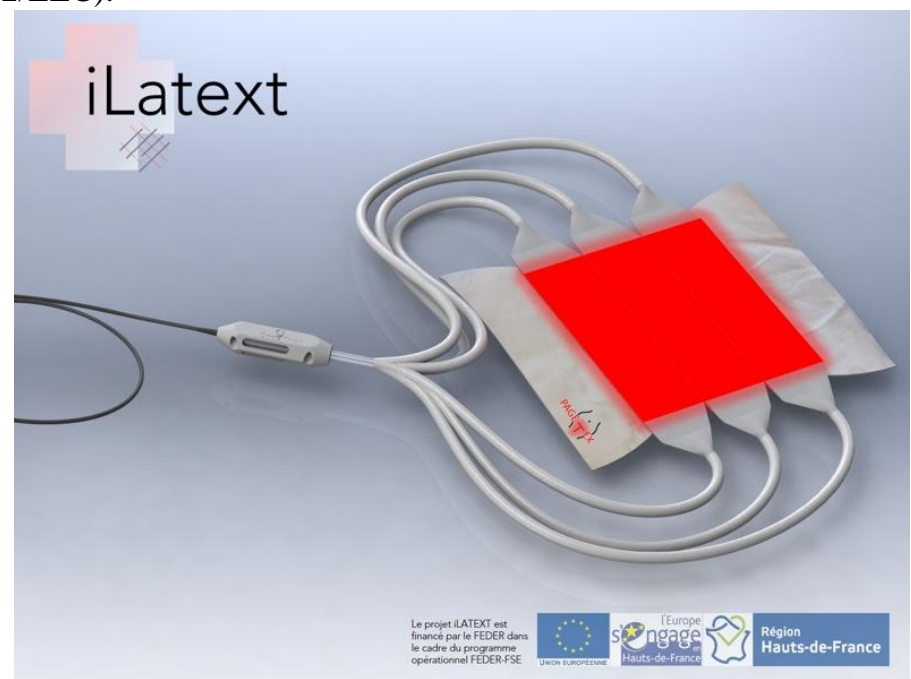

Figure 1 PAGETEX® device

LEF knitted technology allow to fit the body shapes and provides a homogeneous light at the entry of the vagina, under the lips and on perianal region safely, as it does not provide additional thermal emission [10, 20]. During the PDT treatment, the PAGETEX ${ }^{\circledR}$ device is placed over the vulva and maintained by pants. Patients can even slightly move during the illumination session, and also be accompanied while keeping intimacy.
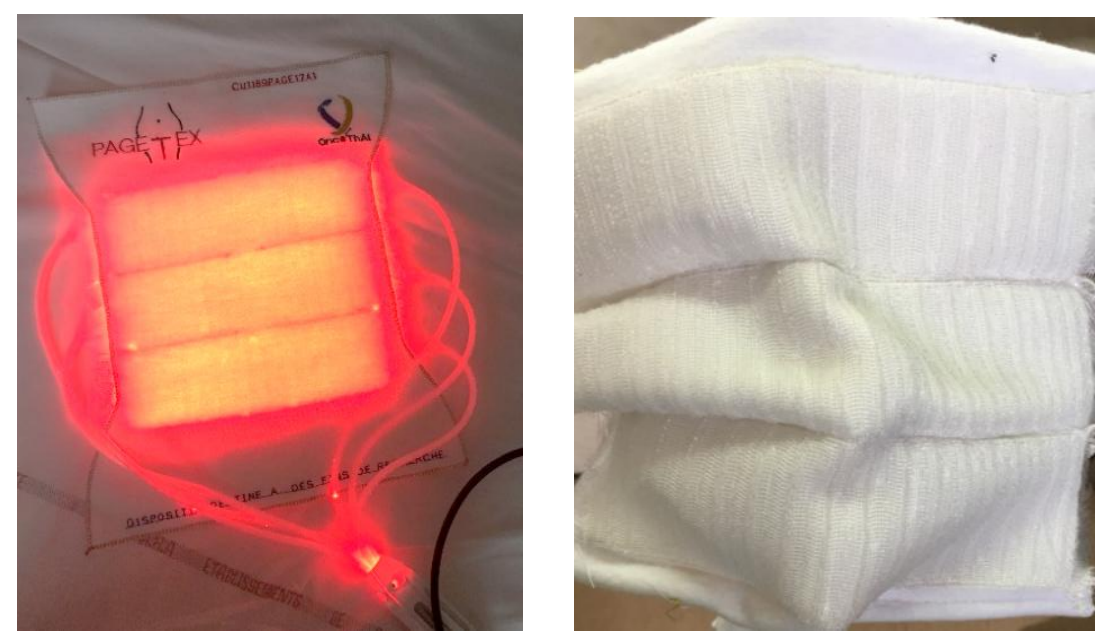

Figure 2 PAGETEX® device connected with 635 nm LASER source - flat device (Left), folded device (Right)

Inspired from the PHOS-ISTOS study light parameters [15], PAGETEX®-PDT involves the application of MAL cream for 30 minutes followed by 2.5 hours of illumination with a fabric-based biophotonic device, without removing MALcream, such that a total light dose of approximately $12 \mathrm{~J} / \mathrm{cm}^{2}$ is delivered.

Transparent occlusive panties keep the biophotonic device completely isolated from the patient's skin and thus to be reusable after specific cleaning.

\subsection{Optical validation}

Irradiance measurements were performed according to the standard NF G20-030 "Optical fiber light textiles Determination of irradiance and luminance of light emitting fabrics", using a positioning template to hold an opto- 
electronic sensor (PD300RM, OPHIR, Israel) and a powermeter (Laserstar, OPHIR, Israel) in 9 holes regularly placed over the effective area.
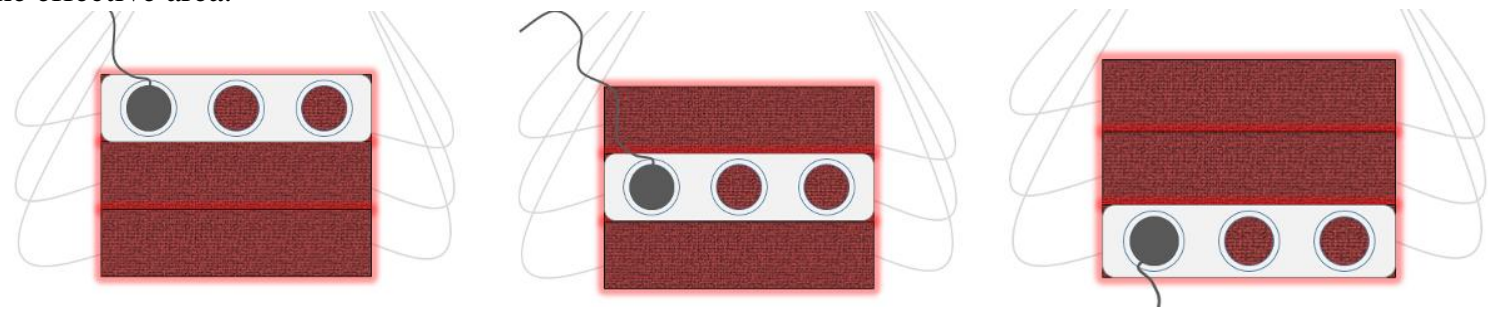

Figure 3 Irradiance measurements

To be declared compliant, the average irradiance values must be between $1.6 \mathrm{~mW} / \mathrm{cm}^{2}$ and $2.4 \mathrm{~mW} / \mathrm{cm}^{2}$ (corresponding to the $20 \%$ maximum variations).

\subsection{PAGETEX® clinical study}

Vulvar EMPD is a rare cancer and PDT is in the process of being extended to other dermatological conditions than skin precancerous lesions. Thus, only few case studies of PDT treatment of vulvar EMPD are available in the literature, and none of them is an efficacy study $[3,4,7,8]$.

The objective of the clinical study is to demonstrate the efficacy of PAGETEX®-PDT on the few eligible patients using quantitative criteria and objective way of analysis. The PAGETEX ${ }^{\circ}$ clinical study (NCT03713203) is currently conducted in the department of dermatology of the Lille University Hospital and aims to establish PAGETEX®-PDT efficacy and tolerability.

\section{CONCLUSION}

PAGETEX® has been developed to overcome the lack of compliant illumination device for the PDT treatment of vulvar EMPD. The device is based on knitted light emitting fabrics technology and is entirely designed to fit with the genital and perianal region. A clinical study is currently ongoing to establish PAGETEX®-PDT efficacy and tolerability, with final results expected for 2023. LEF-based biophotonic device are already used for PDT in dermatology and good clinical outcomes could play a determinant role on the development of a modality that can be clinically useful and really benefit patients with EMPD.

\section{ACKNOWLEDGEMENTS}

This project is supported by the European FEDER funding "iLatext", and is conducted by OncoThAI research lab, in partnership with the company MDB TEXINOV.

\section{REFERENCES}

1. Parker, L.P., et al., Paget's disease of the vulva: pathology, pattern of involvement, and prognosis. Gynecol Oncol, 2000. 77(1): p. 183-9.

2. Kim, S.J., et al., Surgical Treatment and Outcomes of Patients With Extramammary Paget Disease: A Cohort Study. Dermatol Surg, 2017. 43(5): p. 708-714.

3. Raspagliesi, F., et al., Photodynamic therapy using a methyl ester of 5-aminolevulinic acid in recurrent Paget's disease of the vulva: a pilot study. Gynecol Oncol, 2006. 103(2): p. 581-6.

4. Clement, E., et al., [Photodynamic therapy for the treatment of extramammary Paget's disease]. Ann Dermatol Venereol, 2012. 139(2): p. 103-8. 
5. Fontanelli, R., et al., Photodynamic therapy with M-ALA as non surgical treatment option in patients with primary extramammary Paget's disease. Gynecol Oncol, 2013. 130(1): p. 90-4.

6. Louis-Sylvestre, C., B. Haddad, and B.J. Paniel, Paget's disease of the vulva: results of different conservative treatments. Eur J Obstet Gynecol Reprod Biol, 2001. 99(2): p. 253-5.

7. Gao, Y., et al., Efficacy and safety of topical ALA-PDT in the treatment of EMPD. Photodiagnosis Photodyn Ther, 2015. 12(1): p. 92-7.

8. Rioli, D.I., et al., Efficacy and tolerance of photodynamic therapy for vulvar Paget's disease: a multicentric retrospective study. Eur J Dermatol, 2018. 28(3): p. 351-355.

9. Cochrane, C., et al., New design of textile light diffusers for photodynamic therapy. Mater Sci Eng C Mater Biol Appl, 2013. 33(3): p. 1170-5.

10. Mordon, S., et al., Light emitting fabric technologies for photodynamic therapy. Photodiagnosis Photodyn Ther, 2015. 12(1): p. 1-8.

11. Vicentini, C., et al., Treatment of a vulvar Paget's disease by photodynamic therapy with a new light emitting fabric based device. Lasers Surg Med, 2017. 49(2): p. 177-180.

12. Vignion-Dewalle, A.S., et al., A New Light-Emitting, Fabric-Based Device for Photodynamic Therapy of Actinic Keratosis: Protocol for a Randomized, Controlled, Multicenter, IntraIndividual, Phase II Noninferiority Study (the Phosistos Study). JMIR Res Protoc, 2019. 8(4): p. e12990.

13. Morton, C., et al., European Dermatology Forum Guidelines on topical photodynamic therapy. Eur J Dermatol, 2015. 25(4): p. 296-311.

14. Gholam, P., et al., Low irradiance compared with conventional photodynamic therapy in the treatment of actinic keratoses. Photodermatol Photoimmunol Photomed, 2018.

15. Mordon, S., et al., The conventional protocol versus a protocol including illumination with a fabric-based biophotonic device (the Phosistos protocol) in photodynamic therapy for actinic keratosis: a randomized, controlled, non-inferiority clinical study. Br J Dermatol, 2019.

16. Vicentini, C., et al., Photodynamic therapy for actinic keratosis of the forehead and scalp: a randomized, controlled, phase II clinical study evaluating the non-inferiority of a new protocol involving irradiation with a light-emitting, fabric-based device (the Flexitheralight protocol) compared with the conventional protocol involving irradiation with the Aktilite CL 128 lamp. Br J Dermatol, 2018.

17. Vicentini, C., et al., Évaluation de la dose de lumière délivrée au cours du traitement de kératoses actiniques du scalp par photothérapie dynamique. Annales de Dermatologie et de Vénéréologie, 2015. 142(12, Supplement): p. S599-S600.

18. Thecua, E., et al., Light emitting fabric for photodynamic treatment of actinic keratosis. SPIE BiOS. Vol. 10037. 2017: SPIE.

19. Tylcz, J.B., C. Vicentini, and S. Mordon, 4 - Light emitting textiles for a photodynamic therapy, in Smart Textiles and their Applications, V. Koncar, Editor. 2016, Woodhead Publishing: Oxford. p. 71-87. 
20. E. Thecua, C.V., A-S. Vignion, F. Lecomte, P. Deleporte, et al. Light emitting fabric for photodynamic treatment of actinic keratosis. in SPIE BIOS. 2017. San Francisco. 